\title{
Is the Kerr black hole a super accelerator?
}

\author{
S. Krasnikov* \\ Central Astronomical Observatory at Pulkovo, St.Petersburg, 196140, Russia \\ M. V. Skvortsovat \\ Peoples Friendship University of Russia (RUDN University), \\ 6 Miklukho-Maklaya St, Moscow, 117198, Russian Federation
}

\begin{abstract}
A number of long-standing puzzles, such as the origin of extreme-energy cosmic rays, could perhaps be solved if we found a mechanism for effectively transferring energy from black holes to particles and, correspondingly, accelerating the latter to (unboundedly, as long as we neglect the back reaction) large velocities. As of today the only such candidate mechanism in the case of the nonextreme Kerr black hole is colliding a particle that freely falls from infinity with a particle whose trajectory is subject to some special requirements to fulfil which it has to be suitably corrected by auxiliary collisions. In the present paper we prove that - at least when the relevant particles move in the equatorial plane and experience a single correcting collision - this mechanism does not work too. The energy of the final collision becomes unboundedly high only when the energies of the incoming particles do.
\end{abstract}

\section{INTRODUCTION AND CONCLUSION}

Black holes (BHs) are often thought of as one-way membranes and one does not expect them to be sources of high-energy phenomena such as galactic jets or cosmic rays. Penrose [1, 2] was first to find a mechanism enabling one to extract the rotational energy from a Kerr black hole. The idea is based on the fact that near the horizons of these BHs particles may have "negative energies." So, one can split a particle falling in a Kerr BH into two pieces one of which acquires negative energy, while the other escapes to infinity with energy greater then that of the initial particle. Another way of extracting the energy of a rotating

*Electronic address: krasnikov.xxi@gmail.com

${ }^{\dagger}$ Electronic address: milenas577@mail.ru 
$\mathrm{BH}$ is superradiance - wave amplification due to scattering from the black hole - discovered by Zel'dovich and Starobinsky [3, 4]

In response to Wheeler's idea [5] that it is the "energy mining from BHs" that underlies the galactic jets, Bardeen, Press and Teukolsky [6] thoroughly analyzed the Penrose process for stars tidally disrupting near a Kerr black hole. They came to the conclusion that for real astrophysical objects no significant energy gain can be obtained in this process. The same turned out to be true in the general case: as was shown by Wald [7] the energy of the outgoing particle in the above-described reaction is roughly the same as it would be in the Minkowski space.

Shortly thereafter Piran, Shaham and Katz [8] considered the Penrose process in which a particle instead of decaying scatters from another particle. They argued that this new process is much more efficient and that the energy gain grows unboundedly as the $\mathrm{BH}$ approaches the extreme (i. e., the maximally spinning) Kerr solution. The limiting case was considered in 2009 by Banados, Silk, and West (BSW), who found a pole in the expression for the energy of a two-particle collision in a vicinity of an extreme Kerr BH [9]. Their discovery meant that such BHs work as super accelerators, making particles collide with unboundedly large energies. The objections against this process [10, 11] reduced to the following: (1) black holes described by the extreme Kerr solution presumably do not exist in nature. At the same time the energy gain increases exceedingly slowly as the maximally spinning case is approached, so the extreme Kerr is a nonadequate description of a realistic BH; (2) high energy collisions take place very close to the horizon and the time needed for the outgoing particle to reach a distant observer outside the $\mathrm{BH}^{1}$ is of cosmological scale.

What gave reason to hope that the listed objections could be got around was the factdiscovered by Grib and Pavlov (GP) in their well-known paper [12], see also [13], - - that there are poles similar to BSW's in the non-extreme case, too. The problem, however, is that there is a "potential barrier" that does not let particles falling from infinity with suitable parameters (energy and angular momentum) approach the horizon and collide. The way out proposed by GP (for some generalizations see [14 16] and references therein) was to employ additional collisions which would slightly change the parameters of one of the incoming

\footnotetext{
${ }^{1}$ This particle is highly red-shifted due to the energy loss in the gravitational field of the BH. However, this does not compromise the $\mathrm{BH}$ as super collider: such a particle can still be of interest being an outcome of a superhigh-energy reaction.
} 
particles, $\alpha$, and enable it to reach the target, the particle $\mu$, in the desired point (see the next section for details). This scenario (complemented with some conjecture on the properties of multiple scattering in accretion disks, see [12]) has been well received by the community [17, 18] and seemed to settle the whole problem.

To summarize, at present the question of whether (nonextreme Kerr) black holes can generate unboundedly high-energy ${ }^{2}$ particle collisions reduces essentially to the question of whether the above mentioned mechanism is feasible. In this paper we argue that - in its simplest variant - it is not: for particles with bounded energy at infinity the collision energy remains bounded too (for a rigorous formulation see section 【II).

For the sake of simplicity and definiteness we consider a special case. So, technically speaking one might hope that the unboundedly high acceleration can be achieved by removing the corresponding constraints, i. e., by allowing the particles to leave the equatorial plane or by using a different way of correcting the trajectory of $\alpha$ (in the general case $\alpha$ is affected by a set of particles. They fall into the ergosphere, interact with each other, and some of them hit $\alpha$ (more than once, perhaps). $)^{3}$. Either of these situations differs from ours, but not qualitatively. So, it is hard to imagine why a quantity bounded in the latter case would be unbounded in the former. This strongly suggests that the question in the title must be answered negatively in the general case too. Moreover, we conjecture that the same is true for BHs of any type as long as they are stationary and lack geodesics infinitely approaching the horizon.

\section{THE KERR BLACK HOLE AS A SUPER ACCELERATOR}

Consider a pair of particles freely falling in a rotating black hole. Our subject matter is the energy $E_{\text {c.m. }}$. of these particles' collision as measured in their centre of mass system. Can the black hole be a "super accelerator," i. e. can $E_{\text {c.m. }}$ turn out to be unboundedly large, given initially, in the asymptotically flat region $r \rightarrow \infty$, the particles are (almost) at rest in an appropriate sense? At first glance the answer is negative: it is conceivable that $E_{\text {c.m. }}$ unboundedly grows as some parameter $\varsigma$ tends to its limiting value $\varsigma_{0}$, but then what

\footnotetext{
${ }^{2}$ Not to be confused with very high-energy.

${ }^{3}$ Yet another option is to abandon auxiliary collisions and instead to allow $\alpha$ to decay into two lighter particles [19]. This, though, reduces to our scenario with $m_{2}=0$.
} 
happens at $\varsigma=\varsigma_{0}$ looks puzzling. Surprisingly, the correct answer may be positive. This was discovered by Banados, Silk, and West [9] who considered the situation when the black hole in discussion is described by the extreme Kerr solution, which is the case $a=M$ of the spacetime [20, Section 54]

$$
\begin{aligned}
& \mathrm{d} s^{2}=\mathrm{d} t^{2}-\frac{2 M r\left(\mathrm{~d} t-a \sin ^{2} \theta \mathrm{d} \varphi\right)^{2}}{r^{2}+a^{2} \cos ^{2} \theta} \\
&-\left(r^{2}+a^{2} \cos ^{2} \theta\right)\left(\frac{d r^{2}}{\Delta}+d \theta^{2}\right)-\left(r^{2}+a^{2}\right) \sin ^{2} \theta d \varphi^{2}
\end{aligned}
$$

(here

$$
\Delta \equiv r^{2}-2 M r+a^{2}
$$

while $M$ and $a$ are parameters called the mass and specific angular momentum of the space). In this spacetime the distance between the event horizon

$$
r=r_{H} \equiv M+\sqrt{M^{2}-a^{2}}
$$

and a point outside $\mathrm{it}^{4}$, is infinite in contrast to the nonextreme case $a<M$. In particular, there are timelike geodesics outside the horizon that approach the latter but never reach it (loosely speaking, the corresponding particle falls towards the horizon for an infinitely long time, no wonder it gains an infinitely large energy).

Consider a massive particle freely falling in the $r>r_{H}$ part of the Kerr black hole. Let this particle move in the equatorial plane (from now on the same is required from all particles under consideration). Then it moves on a timelike geodesic $\alpha \equiv(t(\tau), r(\tau), 0, \phi(\tau))$, where $\tau$ stands for the proper time of the particle, obeying the conditions [20, Section 61] that

$$
\varepsilon \text { and } L \text { are constant along } \alpha \text {, }
$$

where

$$
\begin{gathered}
\varepsilon \equiv g\left(\partial_{t}, \partial_{\tau}\right)=\dot{t}\left(1-\frac{2 M}{r}\right)+\dot{\phi} \frac{2 a M}{r} \\
L \equiv-g\left(\partial_{\phi}, \partial_{\tau}\right)=-\frac{2 a M}{r} \dot{t}+\left[\left(r^{2}+a^{2}\right)+\frac{2 a^{2} M}{r}\right] \dot{\phi}
\end{gathered}
$$

and that

$$
(r \dot{r})^{2}=U(\varepsilon, L ; r) \equiv \varepsilon^{2} r^{2}+\frac{2 M}{r}(a \varepsilon-L)^{2}+a^{2} \varepsilon^{2}-L^{2}-\Delta,
$$

where $\equiv \frac{\mathrm{d}}{\mathrm{d} \tau}$.

\footnotetext{
${ }^{4}$ Here by "distance" the quantity is understood defined, say, as $\int_{r_{H}}^{r(s)} \sqrt{g_{r r}(r)} \mathrm{d} r$, where $s$ is the mentioned point.
} 
Remark 1. $\varepsilon$ and $L$ can be viewed as characteristics (functions) of a vector ( $\partial_{\tau}$ in this case), but also, due to (2), as characteristics of the geodesic, $\alpha$, to which that vector is tangent. In the latter case they are called, respectively, the specific total energy (at infinity) and the angular momentum of the geodesic. The coordinates of a particle at some particular $\tau_{0}$ in combination with relations (3) fully determine the trajectory of the particle. In particular, the function $U$ may serve as an indicator of accessibility: eq. (3c) implies that a particle with $L=L_{0}$ and $\varepsilon=\varepsilon_{0}$ cannot find itself at a point with $r=r_{0}$ if $U\left(\varepsilon_{0}, L_{0} ; r_{0}\right)<0$.

Consider a collision at a point $o$ of two particles of masses $m_{\alpha}$ and $m_{\mu}$. Let their world lines before $o$ be certain geodesics $\alpha$ and $\mu$. In the center of mass system the energy $E_{\text {c.m. }}$. of the collision is [21]

$$
E_{\mathrm{c} . \mathrm{m} .}^{2}(\alpha, \mu ; o)=m_{\alpha}^{2}+m_{\mu}^{2}+2 m_{\alpha} m_{\mu} g(\dot{\alpha}(o), \dot{\mu}(o))
$$

where $\dot{\alpha}$ and $\dot{\mu}$ are the velocities at $o$ of $\alpha$ and $\mu$, respectively, while $g(\cdot, \cdot)$ is the scalar product $g(\mathbf{a}, \mathbf{b}) \equiv a_{i} b^{i} . E_{\text {c.m. }}$ is a local quantity, so we could alternatively write $E_{\text {c.m. }}^{2}(\dot{\mu}, \dot{\alpha} ; o)$, cf. remark 1.

What BSW discovered was a pole in the expression (44) in the case when particles that initially (at infinity) were at rest, collide at the horizon of an extreme Kerr BH. However, when a black hole is nonextreme any body reaches the horizon within a finite (proper) time and one does not expect any infinity to appear as a result of this process. So, it came as a great surprise when Grib and Pavlov discovered [12] that $E_{\text {c.m. }}$ has poles even in the nonextreme case. [19, Eqs. 11,13] say (in our notation)

$$
\begin{gathered}
\frac{E_{\mathrm{c} . \mathrm{m} .}^{2}(\alpha, \mu ; o)}{2 m_{\mu} m_{\alpha}}=\frac{m_{\mu}^{2}+m_{\alpha}^{2}}{2 m_{\mu} m_{\alpha}}-\varepsilon_{\mu} \varepsilon_{\alpha}+\frac{1}{r \Delta}\left[L_{\mu} L_{\alpha}(2 M-r)+2 \varepsilon_{\mu} \varepsilon_{\alpha}\left(r^{2}(r-M)+a^{2}(r+M)-a M\left(\frac{L_{\mu}}{\varepsilon_{\mu}}+\frac{L_{\alpha}}{\varepsilon_{\alpha}}\right)\right)\right. \\
-M \sqrt{2 \varepsilon_{\mu}^{2} r^{2}+2\left(L_{\mu}-\varepsilon_{\mu} a\right)^{2}-L_{\mu}^{2} r / M+\left(\varepsilon_{\mu}^{2}-1\right) r \Delta / M^{2}} \\
\left.\times \sqrt{2 \varepsilon_{\alpha}^{2} r^{2}+2\left(L_{\alpha}-\varepsilon_{\alpha} a\right)^{2}-L_{\alpha}^{2} r / M+\left(\varepsilon_{\alpha}^{2}-1\right) r \Delta / M^{2}}\right] \quad(5)
\end{gathered}
$$

whence according to [19]

$$
\begin{aligned}
\left.E_{\mathrm{c.m} .}^{2}\right|_{r=r_{H}}=m_{\alpha}^{2}+m_{\mu}^{2} & -\frac{m_{\alpha} m_{\mu}}{2 M^{2}} L_{\alpha H} L_{\mu H} \\
+ & \frac{m_{\alpha} m_{\mu}}{4 M^{2}}\left[\left(L_{\alpha H}^{2}+4 M^{2}\right) \frac{L_{\mu H}-L_{\mu}}{L_{\alpha H}-L_{\alpha}}+\left(L_{\mu H}^{2}+4 M^{2}\right) \frac{L_{\alpha H}-L_{\alpha}}{L_{\mu H}-L_{\mu}}\right],
\end{aligned}
$$


where

$$
L_{\varpi H}\left(\varepsilon_{\varpi}\right) \equiv\left(2 r_{H} M / a\right) \varepsilon_{\varpi}, \quad \varpi=\alpha, \mu,
$$

and $\varepsilon_{\varpi}$ is the specific total energy of the particle $\varpi$. A pair of parameters $\varepsilon$ and $L$ related by the equality $L=\left(2 r_{H} M / a\right) \varepsilon$ is termed critical. It follows from (6) that the energy of a two-particle collision near the horizon of a Kerr black hole is arbitrarily large if a particle that has $L=L_{1} \neq L_{1 H}$ collides with a particle whose parameters are sufficiently close to critical.

Warning. In considering particle mechanics on the Kerr background one should be particularly careful with the word "energy." If we pick a unit timelike vector $\boldsymbol{T}$ at a point $p$ of a geodesic $\alpha(\tau)$, then the quantity $\mathcal{E}_{\boldsymbol{T}}(\alpha) \equiv m g(\boldsymbol{T}, \dot{\alpha})$ is the energy at $p$ of the particle of mass $m$ moving on $\alpha$ as measured by the observer who is located at $p$ and whose 4 -velocity at that point is $\boldsymbol{T}$. This notion is closely related to the "energy of collision" $E_{\text {c.m. }}$ appearing in (4). But there is also the "energy at infinity" $m \varepsilon(\alpha)$ of a particle $\alpha$. The BSW and GP effects show that particles of equal energies $\varepsilon$ may have most different energies $\mathcal{E}_{\boldsymbol{T}}$.

For future use note that when

$$
\varepsilon_{\mu}, L_{\mu}, \text { are constant, } \quad \varepsilon_{\alpha}, L_{\alpha} \text { tend to constants, } \quad L_{\mu} \neq L_{\mu H}\left(\varepsilon_{\mu}\right)
$$

and

$$
\frac{L_{\alpha}}{\varepsilon_{\alpha}}=\frac{L_{\alpha H}}{\varepsilon}+\varphi, \quad r(o)=r_{H}+\xi,
$$

where $L_{\alpha H}, \varepsilon$ is a critical pair and $\varphi$, and $\xi$ are infinitesimal, the expression in the square brackets in (5) divided by $\varepsilon_{\mu} \varepsilon_{\alpha}$ has the form

$$
A+\xi B+\varphi C-\sqrt{D_{1}+\xi E_{1}+O\left(\xi^{2}\right)} \sqrt{D_{2}+\xi E_{2}+\varphi F+G \varphi^{2}+o(\xi)}+o(\xi),
$$

where the uppercase letters are some constants. Comparing this with (6), which says that for some constant $\Phi,\left.E_{\text {c.m. }}^{2}\right|_{\xi \rightarrow 0} \sim \Phi \varphi^{-1}$, we infer that

$$
A, D_{2}, F=0, \quad D_{1}, E_{2}, G \neq 0, \quad D_{1} G=C^{2}
$$

Consequently, in the leading order the expression (9) is equal to

$$
\begin{cases}\frac{E_{2}}{2 \sqrt{G}} \xi \varphi^{-1}, & \text { at } \xi=o\left(\varphi^{2}\right) \\ {\left[C-\sqrt{D_{1}\left(E_{2}+G\right)}\right] \sqrt{\xi},} & \text { at } \varphi \sim \sqrt{\xi} \\ \sqrt{D_{1} E_{2}} \sqrt{\xi}, & \text { at } \varphi=o(\sqrt{\xi})\end{cases}
$$




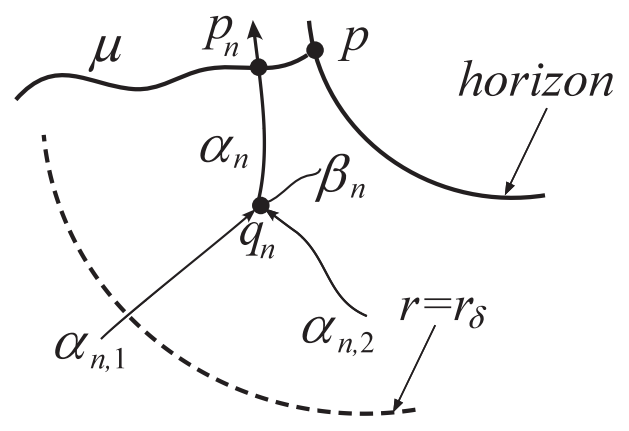

a)

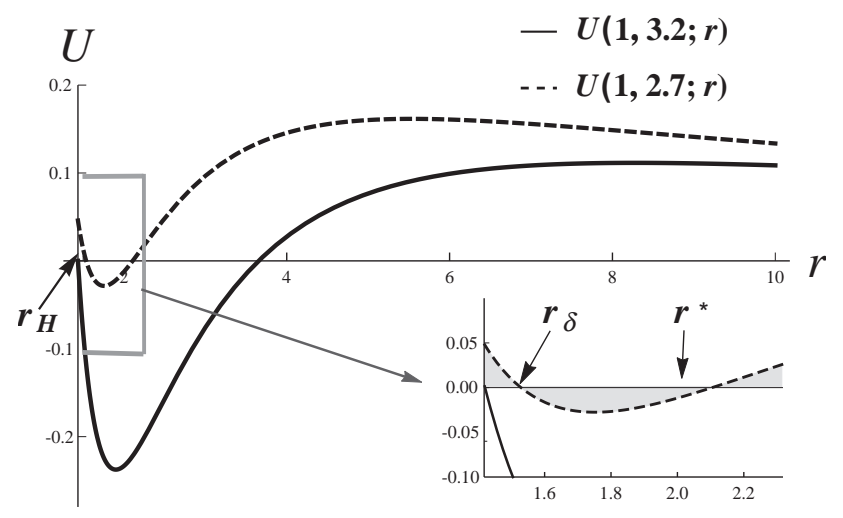

b)

FIG. 1: a) $\alpha_{n, 1}$ and $\alpha_{n, 2}$ come from infinity to collide at $q_{n}: \alpha_{n, 1}+\alpha_{n, 2} \rightarrow \alpha_{n}+\beta_{n}$. One of them collide then with $\mu$ at $p_{n}$. b) The function $U(\varepsilon, L ; r)$ for $M=1, a=0.9$. In the case of the solid line the parameters are critical.

[that the coefficient in the square brackets (in the second line) is non-zero is seen from (10)]. It follows that

conditions (8a $18 \mathrm{~b})$ imply the divergence $E_{\text {c.m. }}(\alpha, \mu ; o) \rightarrow \infty$,

(which does not follow automatically from (66) the latter being applicable only to the case $\xi=o(\varphi))$.

Equation (6) does not yet make the black hole a super accelerator: it may happen that for some reason particles cannot both come from the asymptotically flat region. And we shall see that this is the case indeed. Let $L_{\delta}, \varepsilon_{\delta}$ be functions of $\delta$ such that:

$$
\lim _{\delta \rightarrow 0} \varepsilon_{\delta}=\varepsilon, \quad \lim _{\delta \rightarrow 0} L_{\delta}=L_{H}-0,
$$

where $L_{H}$ and $\varepsilon$ is a critical pair. Then ${ }^{5}$ there exists a function $r_{\delta}>r_{H}$ such that:

$$
\begin{gathered}
U(\varepsilon, L ; r) \text { is non-negative on }\left[r_{H}, r_{\delta}\right] \text { and changes to negative at } r=r_{\delta} \\
\lim _{\delta \rightarrow 0} r_{\delta}=r_{H}
\end{gathered}
$$

It follows, in particular ${ }^{6}$ that particles freely falling from infinity cannot take the position necessary for colliding with unbounded $E_{\text {c.m. }}$ : the annulus $\left[r_{H}, r_{\delta}(L)\right]$ that must be entered by the particles is separated from the infinity by the "potential barrier" $\{U(L)<0\}$.

\footnotetext{
${ }^{5}$ See, for example, 21, eq. (25)], in which, according to [22], $x_{\delta}$ is $\frac{1}{M} \times\left(\right.$ what we denote by $\left.r_{\delta}\right)$.

${ }^{6}$ For a rigorous derivation one also must take into consideration that there are no circular geodesics near the horizon, see the proof of the lemma.
} 
To solve this problem in the spirit of GP's "auxiliary collision" proposal we shall use pairs of particles: two particles, $\alpha_{n, 1}$ and $\alpha_{n, 2}$, see figure 1a, are tossed into the ergosphere. They collide in a point $q_{n}$ near the horizon and as a result one of them transforms into the particle moving on the geodesic $\alpha_{n}$ with desired values of the parameters $\varepsilon\left(\alpha_{n}\right)$ and $L\left(\alpha_{n}\right) \approx L_{H}\left(\varepsilon\left(\alpha_{n}\right)\right)$.

Though this process leads to unboundedly large $E_{\text {c.m. }}$ it seems plausible since the difference $L\left(\alpha_{n, 1}\right)-L_{H}\left(\alpha_{n, 1}\right)$ before the collision can be made quite small, see [12]. This argument, however, is not decisive. It is conceivable, for example, that the proposed solution suffers exactly the same problem as the initial scenario: to properly correct at a point $x$ the trajectory of particle 1 the second particle must have parameters $\varepsilon$ and $L$ incompatible with the fact that it came to $x$ from the region outside the static limit.

In the next section we show that this is the case and the above described process does not allow one to unboundedly accelerate particles.

Remark 2. Loosely speaking, our result means that the effectiveness $K$ of the nonextreme Kerr black hole as particle accelerator is bounded for any particular energy:

$$
\text { for any } \varepsilon \quad K(\varepsilon) \equiv \max \frac{E_{\text {c.m. }}(\varepsilon)}{\varepsilon}<\infty, \quad \varepsilon \equiv \varepsilon\left(\alpha_{1}\right)+\varepsilon\left(\alpha_{2}\right) \text {. }
$$

There is, however, another problem, which is closely related to the former one, but which is too hard to be considered in this paper ${ }^{7}$. Specifically, it might be interesting to find exactly how large $K$ is. In principle, one can imagine that $\varepsilon=o\left(E_{\text {c.m. }}\right)$, when $\varepsilon \rightarrow \infty$, i. e., that $K$ of the nonextreme Kerr black holes grows unboundedly with the incoming energy. This would make such black holes "almost super accelerators," a phenomenon not yet considered in the literature (to our knowledge).

\section{UNBOUNDED ACCELERATION}

Consider a sequence of elastic collisions realizing our scenario of obtaining a diverging sequence of $E_{\text {c.m. }}$. Geometrically speaking, that is a set of points of the nonextreme Kerr spacetime

$$
q_{n}: \quad n \in \mathbb{N}, \quad \lim _{n \rightarrow \infty} r\left(q_{n}\right)=r_{H}
$$

\footnotetext{
${ }^{7}$ We are grateful to the anonymous referee for drawing our attention to this problem.
} 
and timelike future directed geodesic segments $\mu, \alpha_{n, 1}, \alpha_{n, 2}, \alpha_{n}, \beta_{n}$-see figure 1 h-such that:

I. For each $n$ the geodesics $\alpha_{n, 1}, \alpha_{n, 2}$ start at the infinity $r=\infty$ and terminate at the point $q_{n}$, where the geodesics $\alpha_{n}$ and $\beta_{n}$ start. The particles $\alpha_{n, 1}$ and $\alpha_{n, 2}$ have masses $m_{1}$ and $m_{2}$, while the masses of the particles moving on $\alpha_{n}$ and $\beta_{n}$ are $m_{3}$ and $m_{4}$, respectively. The geodesics are related by the equality

$$
m_{1} \dot{\alpha}_{n, 1}+\left.m_{2} \dot{\alpha}_{n, 2}\right|_{q_{n}}=m_{3} \dot{\alpha}_{n}+m_{4} \dot{\beta}_{n}
$$

(energy-momentum conservation).

II. The geodesic $\mu$ is noncritical, i. e.,

$$
L(\mu)=L_{\mu} \neq L_{\mu H}
$$

and it is the collision of $\mu$ with $\alpha_{n}$ that occurs with unboundedly high $E_{\text {c.m. }}$ :

$$
\lim _{n \rightarrow \infty} g\left(\boldsymbol{v}_{n}, \dot{\alpha}_{n}\left(p_{n}\right)\right)=\infty .
$$

Here $L_{\mu}$ is some constant and $\boldsymbol{v}_{n}$ at each $n$ is the vector of $T_{p_{n}}$ tangent to the geodesic obtained from $\mu$ by a combination of isometries $\phi \rightarrow \phi+c_{1}$ and $t \rightarrow t+c_{2}$, so all $\boldsymbol{v}_{n}$ have the same $\varepsilon$ and $L$.

Let us show that in spite of (15) the particles under discussion are not (unboundedly) accelerated by a nonextreme black hole: the diverging $E_{\text {c.m. }}$ implies the diverging energies of the incoming particles $\alpha_{n, i}$.

Assertion. The sequence $\left\{\alpha_{n, i}\right\}$ contains a subsequence $\left\{\alpha_{k, i_{0}}\right\}, i_{0} \equiv 1$ or $i_{0} \equiv 2$, with unboundedly large energies at infinity:

$$
\lim _{k \rightarrow \infty} \varepsilon_{k, i_{0}}=+\infty
$$

(from now on we write $L_{n, i}, \varepsilon_{n, i}$ for $L\left(\alpha_{n, i}\right), \varepsilon\left(\alpha_{n, i}\right)$ and $L_{n}, \varepsilon_{n}$ for $L\left(\alpha_{n}\right), \varepsilon\left(\alpha_{n}\right)$ ).

We begin with establishing an auxiliary - purely algebraic - property of $U$.

Lemma. For any pair of critical parameters $\varepsilon^{*}$ and $L^{*}$ there is a constant $r^{*}>r_{H}$ such that

$$
\left.U\left(\varepsilon^{*}, L^{*} ; r\right)\right|_{\left(r_{H}, r^{*}\right)}<0 .
$$

(which means, in particular, that no critical geodesic connects the infinity to the horizon). 
Proof $^{8}$. By (12), there is an $r_{\delta}$ (converging to $r_{H}$ when $\delta \rightarrow 0$ ) such that for all sufficiently small positive constants $\delta$ the function $U\left(\varepsilon^{*}, L^{*}-\delta ; r\right)$ is non-negative at $r_{H} \leq r \leq r_{\delta}$ and changes its sign at $r_{\delta}$. Hence,

$$
U\left(\varepsilon^{*}, L^{*} ; r_{H}\right)=\lim _{\delta \rightarrow 0} U\left(\varepsilon^{*}, L^{*}-\delta ; r_{\delta}\right)=0, \quad U^{\prime}\left(\varepsilon^{*}, L^{*} ; r_{H}\right)=\lim _{\delta \rightarrow 0} U^{\prime}\left(\varepsilon^{*}, L^{*}-\delta ; r_{\delta}\right) \leq 0
$$

where the prime denotes the derivative by $r$. But if (17) does not hold, $U$ cannot satisfy the system $U\left(r_{H}\right)=U^{\prime}\left(r_{H}\right)=0$, because this would mean that there is a circular orbit [6] at $r=r_{H}$, while such orbits are known to be lacking [20] in the nonextreme Kerr black hole. So, $U^{\prime}\left(\varepsilon^{*}, L^{*} ; r_{H}\right)<0$, cf. figure 1 b. Being combined with the first equality in (18) this proves the lemma.

Proof of the assertion. To derive a contradiction assume that the set $\left\{\varepsilon_{n, i}\right\} i=1,2, n=$ $1,2 \ldots$, is bounded. Then the set $\left\{L_{n, i}\right\}$ is bounded too, because at sufficiently large $L, U$ becomes negative [see (3c), at points with $r>2 M]$ while such points exist on every $\alpha_{n, i}$ by its very definition. This boundedness implies that for either $i$ there must be subsequences $\left\{\varepsilon_{k, i}\right\}$ and $\left\{L_{k, i}\right\}$ converging to some $\bar{\varepsilon}_{i}$ and $\bar{L}_{i}$, respectively. Likewise, $\left\{\varepsilon_{k}\right\}$, which is the set of the energies of $\left\{\alpha_{k}\right\}$, is bounded by $\bar{\varepsilon}_{1}+\bar{\varepsilon}_{2}$ and therefore we shall assume without loss of generality that $\left\{\varepsilon_{k}\right\}$ and $\left\{L_{k}\right\}$ converge to some $\bar{\varepsilon}$ and $\bar{L}$.

By continuity

$$
U\left(\bar{\varepsilon}_{i}, \bar{L}_{i} ; r\right)=\lim _{k \rightarrow \infty} U\left(\varepsilon_{k, i}, L_{k, i} ; r\right) .
$$

But the existence of the geodesics $\alpha_{k, i}$ means that

$$
\left.U\left(\varepsilon_{k, i}, L_{k, i} ; r\right)\right|_{\left[r\left(q_{k}\right), \infty\right)} \geq 0
$$

and we conclude [recall that, by $(\underline{12 \mathrm{~b}}), r\left(q_{k}\right) \rightarrow r_{H}$ ] that

$$
\left.U\left(\bar{\varepsilon}_{i}, \bar{L}_{i} ; r\right)\right|_{\left[r_{H}, \infty\right)} \geq 0
$$

This contradicts the lemma and thus completes the proof if the parameters $\bar{\varepsilon}_{i}$ and $\bar{L}_{i}$ are critical.

\footnotetext{
${ }^{8}$ One can prove the lemma by simply substituting the definition (7) into the definition of $U$, but the relevant manipulations are rather formidable.
} 
To prove that this is the case multiply (13) by $\boldsymbol{v}_{k}$ to obtain, with the use of (15)

$$
m_{1} g\left(\boldsymbol{v}_{k}, \dot{\alpha}_{k, 1}\left(q_{k}\right)\right)+m_{2} g\left(\boldsymbol{v}_{k}, \dot{\alpha}_{k, 2}\left(q_{k}\right)\right) \geq m_{3} g\left(\boldsymbol{v}_{k}, \dot{\alpha}_{k}\left(q_{k}\right)\right)
$$

By (41), the right hand side of (19) equals

$$
\frac{1}{2 m_{\mu}}\left[E_{\mathrm{c} . \mathrm{m} .}^{2}\left(\alpha_{k}, \mu ; r\left(q_{k}\right)\right)-m_{3}^{2}-m_{\mu}^{2}\right]
$$

To find the limit of the first term in this expression, note that we come to the situation of (8) by mere renaming

$$
q_{k}=o, \quad \varepsilon_{k}=\varepsilon_{\alpha}, \quad \bar{\varepsilon}=\varepsilon, \quad L_{k}=L_{\alpha}, \quad \bar{L}=L_{\alpha H}
$$

Hence, by (11), the just mentioned term diverges [it is just to replace $E_{\text {c.m. }}\left(r\left(q_{k}\right)\right) \leftrightarrow$ $E_{\text {c.m. }}\left(r\left(p_{k}\right)\right)$ that we need the statement (8) $]$. Correspondingly, the left hand side of (19) diverges too and for $i_{0} \equiv 1$ or for $i_{0} \equiv 2$

$$
g\left(\boldsymbol{v}_{k}, \dot{\alpha}_{k, i_{0}}\left(q_{k}\right)\right) \underset{k \rightarrow \infty}{\longrightarrow} \infty
$$

which implies that

$$
E_{\text {c.m. }}\left(\boldsymbol{v}_{k}, \dot{\alpha}_{k, i_{0}}\left(q_{k}\right)\right) \underset{k \rightarrow \infty}{\longrightarrow} \infty \text {. }
$$

Thus $E_{\text {c.m. }}$ has a pole in $\varepsilon=\bar{\varepsilon}_{i_{0}}, L=\bar{L}_{i_{0}}$ (recall that $\bar{\varepsilon}_{i}$ and $\bar{L}_{i}$ were defined as the limits of $\left\{\varepsilon_{k, i}\right\}$ and $\left\{L_{k, i}\right\}$, respectively). And from (6) it is easy to see that - due to (14) - in the poles of $E_{\text {c.m. }}$ the parameters $\varepsilon$ and $L$ are critical indeed.

\section{Acknowledgements}

We appreciate kind assistance of Kirill A. Bronnikov. The publication has been prepared with the support of the "RUDN University Program 5-100". S. K. is grateful to RFBR for financial support under grant No. 18-02-00461 "Rotating black holes as the sources of particles with high energy."

[1] R. Penrose, reprinted in GRG 34, 1141 (2002).

[2] R. Penrose and R. M. Floyd, Nature Physical Science 229, 177 (1971). 
[3] Ya. B. Zel'dovich, Sov. Phys.-JETP 35, 1085 (1972).

[4] A. A. Starobinsky, Sov. Phys.-JETP 37, 28 (1973).

[5] J. Wheeler, in Study Week on Nuclei of Galaxies, edited by. D. J. K. O'Connell ( Elsevier, New York, 1971)

[6] J. M. Bardeen, W. H. Press, and S. A. Teukolsky, Astrophys. J. 178, 347 (1972).

[7] R. M. Wald, Astrophys. J. 191, 231 (1974).

[8] T. Piran, J. Shaham, and J. Katz, Astrophys. J. 196, L107 (1975).

[9] M. Banados, J. Silk, and S. M. West, Phys. Rev. Lett. 103, 111102 (2009).

[10] K. S. Thorne, Astrophys. J. 191, 507 (1974).

[11] T. Jacobson and T. Sotiriou, Phys. Rev. Lett. 104, 021101 (2010).

[12] A. A. Grib and Yu. V. Pavlov, Astropart. Phys. 34, 581 (2011).

[13] S. Gao and C. Zhong, Phys. Rev. D84, 044006 (2011).

[14] O. B. Zaslavskii, Mod. Phys. Lett. A. 28, 1350037 (2013).

[15] O. B. Zaslavskii, Phys.Rev. D82, 083004 (2010).

[16] M. Patil and P. Joshi, Class. Quantum Grav. 28, 235012 (2011).

[17] Y. Zhu, S. F. Wu, Y. X. Liu, and Y. Jiang, Phys. Rev. D84, 043006 (2011).

[18] T. Harada and M. Kimura, Class. Quantum Grav. 31, 243001 (2014).

[19] A. A. Grib and Yu. V. Pavlov, Grav. Cosmol. 17, 42 (2011).

[20] S. Chandrasekhar, The Mathematical Theory of Black Holes (Oxford University Press, New York, 1983).

[21] A. A. Grib, Yu. V. Pavlov, and O. F. Piattella, Grav. Cosmol. 18, 70 (2012).

[22] Yu. V. Pavlov, (private communication). 